\title{
Small Bowel Obstruction in Intellectual Impairment-Time to Revisit: Case Report
}

\section{Takako Eva Yabe ${ }^{*}$, Suren Subramaniam and Bruce Ashford}

Department of General Surgery, Wollongong Hospital, New South Wales, Australia

"Corresponding author: Takako Eva Yabe, Department of General Surgery, Wollongong Hospital, New South Wales, Australia, Tel: 02-4255-1346; E-mail: takakoyabe424@gmail.com

Received date: October 14, 2017; Accepted date: November 02, 2017; Published date: November 09, 2017

Copyright: $@ 2017$ Yabe TE, et al. This is an open-access article distributed under the terms of the Creative Commons Attribution License, which permits unrestricted use, distribution, and reproduction in any medium, provided the original author and source are credited.

\begin{abstract}
Background: Clinical assessment of people with intellectual disability is challenging to the treating clinicians, mainly because these patients are unable to provide an accurate history of their presenting symptoms. In this report, we describe a case in which we faced a diagnostic dilemma before reaching a decision on definitive management.
\end{abstract}

Case presentation: A 57 year old woman with cognitive impairment from a group home was admitted to hospital with abdominal distension, anorexia and malaise. She had a surgical intervention for trichobezoar many years ago. Her bowel habits were reported to be normal. On examination, she had a distended but non-tender abdomen. A computed tomography (CT) scan showed a small bowel obstruction (SBO). She responded to conservative management and was discharged. She presented a third time 4 weeks later with the same problem. This time, however, she looked lethargic and her biochemistry results were slightly abnormal. A CT scan was performed, which confirmed complete SBO with a clear transition point in the distal small bowel within the pelvis and faecalisation of the small bowel. We decided that, because of her multiple presentations to the hospital with the same symptoms and worsening faecalisation on CT scan, she would benefit from exploratory laparotomy. During surgery, an obstructing mass was identified that was found to be a calcified latex glove. The patient had an uncomplicated postoperative recovery and has not presented to the hospital since then.

Conclusion: A trichobezoar should be suspected and investigated in intellectually impaired patients with gastrointestinal symptoms. Environmental modifications, a neuropsychiatric review and a multidisciplinary approach involving all care-givers should be considered in an effort to avoid this potentially life-threatening complication in a most vulnerable group.

Keywords: Bowel obstruction; Intellectual disability; Foreign body; Trichobezoar

Abbreviations CT: Computed Tomography; SBO: Small Bowel Obstruction

\section{Background}

Small bowel obstruction (SBO) is a life-threatening condition in which the normal enteric flow is impaired due to mechanical blockage of the small intestine [1]. It is most commonly caused by adhesions from previous abdominal surgery, hernia, intussusception or malignancy [2]. Patients with SBO usually present with abdominal pain, distension, nausea, vomiting, constipation and/or obstipation [3]. Bezoars are a well-recognised cause of intraluminal SBO in children and the intellectually impaired [4]. Few cases of bezoar-induced SBO have been reported in the last decade, the most recent series on the subject having been published in 1994 [5]. This may represent a true reduction in incidence, although with the improved general care of this group, we would expect incidence rates to increase. We present what is only the second reported case of a glove used by care providers as the primary reason for the development of a bezoar. Intellectually impaired patients may be more inclined to ingest foreign objects, are often unable to provide a thorough history and can present special challenges during examination. With the significant morbidity and mortality that can complicate such presentations, it is important for treating doctors to be aware of this possible aetiology [6]. In addition, care providers in group homes should be alert to the dangers of swallowing non-digestible foreign bodies.

\section{Case Presentation}

\section{Case description}

A 57 year old woman with cognitive impairment from a group home was admitted to a regional hospital in Australia, New South Wales, with abdominal distension, anorexia and malaise. She had a background of previous laparotomy and enterotomy for a trichobezoar many years ago. Her bowel habits were reported to be normal. She was aphasic and fully dependent on a carer for activities of daily living. On examination, she was haemodynamically stable and afebrile. Her abdomen was distended but soft and non-tender. Biochemistry results were all within normal range. A computed tomography (CT) scan was performed, which showed a possible transition point in her pelvis. As she had previous abdominal surgery in 2004 , her transition point was thought to be due to adhesions. She was admitted under the care of the general surgical team for management of adhesive SBO. She responded to conservative management and was discharged to her group home 3 days later. She presented to us again 2 weeks later with the same symptoms of abdominal distension and poor appetite, but no obvious 
signs of distress from pain. She again responded to conservative management and was discharged to the group home within a few days. She came to the hospital a third time 4 weeks later with the same problem. This time, she looked lethargic and her blood pressure was $143 / 108 \mathrm{~mm} \mathrm{Hg}$, pulse rate 106 beats/min, respiratory rate 17 breaths/ min, and body temperature $36.1^{\circ} \mathrm{C}$. Laboratory evaluation revealed a white blood cell count of $23 \times 10^{9} / \mathrm{L}$ with $89 \%$ neutrophils, a haemoglobin level of $138 \mathrm{~g} / \mathrm{L}$, a platelet count of $536 \times 10^{9} / \mathrm{L}$, and a lactate level of 2.9. Liver and kidney function test results were normal. A CT scan was performed that confirmed complete SBO with a clear transition point in the distal small bowel within the pelvis and faecalisation of the small bowel. She displayed overflow incontinence. We decided that, because of her multiple presentations to the hospital with the same symptoms and worsening faecalisation on the CT scan, she would benefit from an exploratory laparotomy. During surgery, an obstructing mass was identified in the proximal jejunum. An enterotomy was performed and a hardened, semi-calcified foreign body was removed. The enterotomy was closed primarily with a 3-0 polydioxanone suture. Further examination of the specimen showed a mass that consisted of a calcified latex glove with intertwined hair. The patient recovered well post-operatively, regained her appetite and successfully returned to the group home. Following the patient's discharge from hospital, a neuropsychiatric review led to a change in her pharmacotherapy to control pica. The patient's carers were involved in further discussions to prevent recurrences.

\section{Discussion}

Given the role of history taking in the establishment of differential diagnoses in bowel obstruction, the management of patients with a limited verbal capacity is challenging. With life expectancy in this group generally improving, the spectre of recurrent idiopathic bowel obstruction may increase. Even with a contributory history as in this case, arriving at a diagnosis that requires surgical intervention may be difficult and is facilitated by a desire to avoid overtreatment. A strategy to avoid trichobezoars had been implemented for this patient over many years, but it overlooked other potential scenarios, as shown in the eventual operative findings. Four types of bezoar are well recognised: phytobezoars, trichobezoars, pharmacobezoars and lactobezoars [7]. Trichobezoars are defined as gastric concretions of hair fibres typically found in patients with a history of mental illness [8]. In our patient, we found a trichobezoar that consisted of a surgical glove matted with hair. Yakan et al. [9] reported that abdominal pain (49-100\%), epigastric distress (80\%), nausea and vomiting (35-75\%) and SBO (94\%) are the main clinical symptoms of phytobezoars [9]. Some of these signs and symptoms can be difficult to elicit from a patient with cognitive impairment. The CT appearance of a gastrointestinal bezoar - an intraluminal mass containing a mottled air pattern - is characteristic and reported to be a good indicator of bezoars [10]. In our case, the radiologic interpretation was of an intraluminal mass containing gas in the mid-jejunum, which was considered faecalisation in a setting of chronic sub-acute obstruction (Figures 1 and 2).

This result corresponded to the intraoperative findings (Figures 3 and 4). The mass was not detected on the CT scan, which was performed at the patient's first presentation. Most SBOs that occur in patients who have undergone surgery are attributable to adhesions and are primarily treated conservatively, surgery being considered only if other treatment fails or if there is pain.

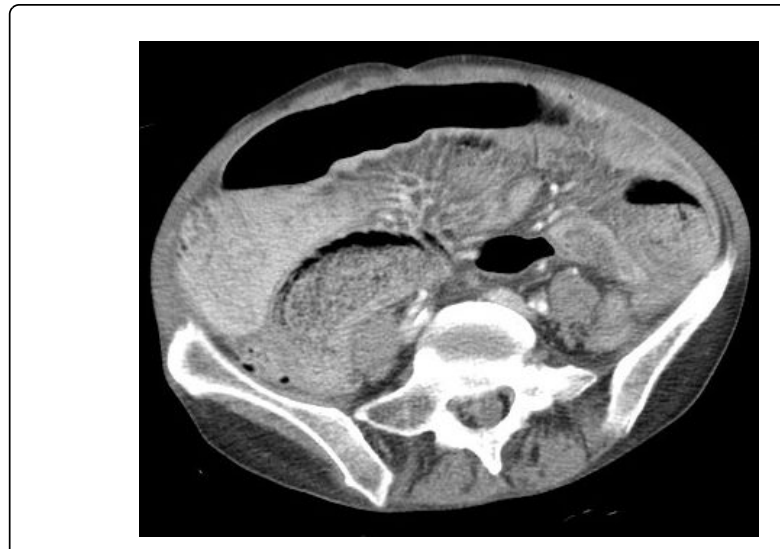

Figure 1: Axial CT of abdomen.

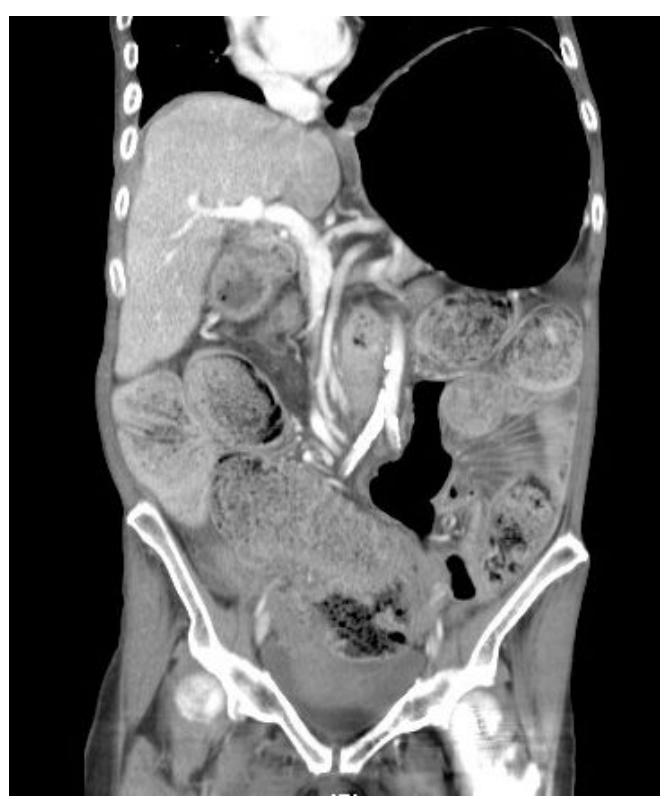

Figure 2: Coronal CT of abdomen.

In this case, the patient had previously undergone a laparotomy, had been managed non-operatively and had responded appropriately during each hospital admission. This transient responsiveness of the patient to non-operative management and the difficulty in communication contributed to a delay in definitive management. Management of underlying disorders is the best approach to prevention. Such an approach includes environmental modification and formal psychiatric follow-up in patients with psychiatric disorders. In this case, specialist input from neuropsychiatry was sought to manage the patient's trichotillomania after discharge.

Her hair was trimmed to minimise it and her carers at the group home were made aware that objects such as gloves can be swallowed, causing gastrointestinal distress. A combined multidisciplinary approach is the key to preventing such episodes in this group of patients. 
Citation: Yabe TE, Subramaniam S, Ashford B (2017) Small Bowel Obstruction in Intellectual Impairment-Time to Revisit: Case Report. Health Care Current Reviews 5: 212. doi:10.4172/2375-4273.1000212

Page 3 of 3

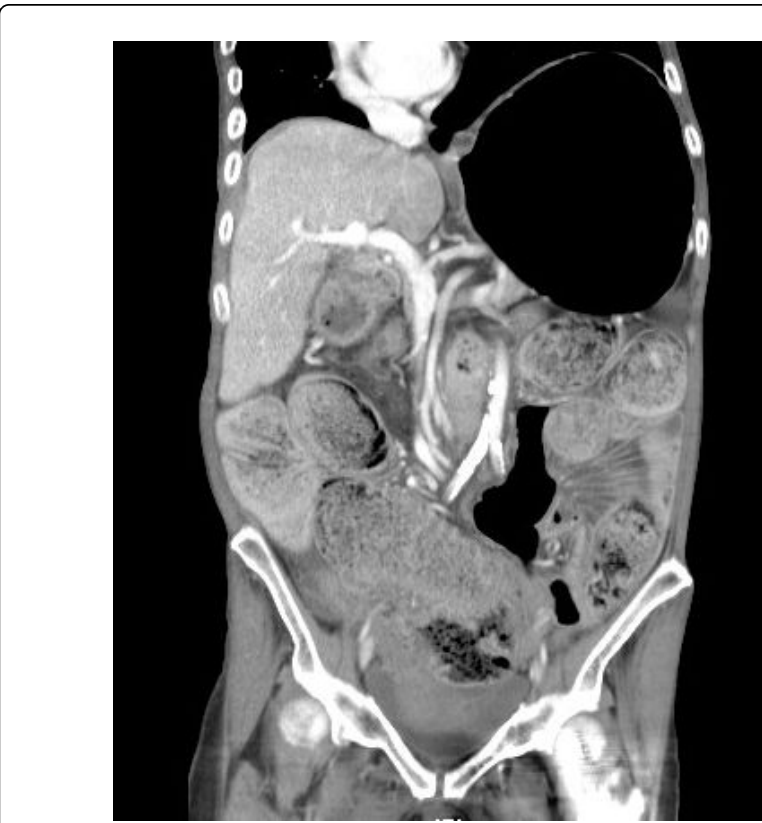

Figure 3: Intraoperative image of mid jejunum.

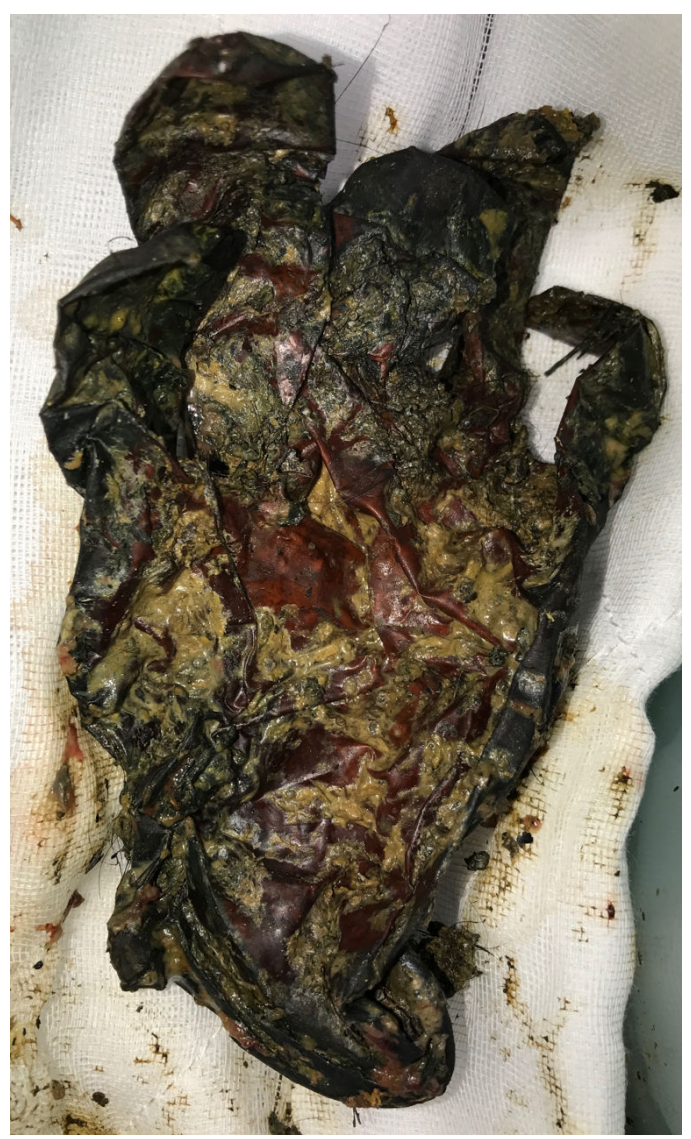

Figure 4: Foreign body.

\section{Conclusion}

Preoperative diagnosis of SBO caused by a bezoar is challenging, but should be suspected and investigated in intellectually impaired patients with gastrointestinal symptoms. The presence of an intraluminal mass may not be easily appreciated on the CT scan, but should be explored if this diagnosis is entertained. Environmental modifications, a neuropsychiatric review and a multidisciplinary approach involving all care-givers should be considered in an effort to avoid this potentially life-threatening complication in a most vulnerable group.

Consent Written informed consent was obtained from the medical power of attorney of the patient for publication of this case report. A copy of the written consent is available for review by the Series Editor of this journal.

\section{Declarations}

\section{Ethics approval and consent to participate and consent to publish}

Written informed consent was obtained from the medical power of attorney of the patient for publication of this case report. A copy of the written consent is available for review by the Series Editor of this journal.

\section{Availability of data and materials}

This case report draws on a single patient's electronic medical record. De-identified data used for this submission will be made available on request to the primary author.

\section{References}

1. Jancar J, Speller C J (1994) Fatal intestinal obstruction in the mentally handicapped. J Intellect Disabil Res 38: 413-422.

2. Silva AC, Pimenta M, Guimaraes LS (2009) Small bowel obstruction: What to look for. Radiographics 29: 423-439.

3. Markogiannakis H, Messaris E, Dardamanis D, Pararas N, Tzertzemelis $\mathrm{D}$, et al. (2007) Acute mechanical bowel obstruction: Clinical presentation, etiology, management and outcome. World J Gastroenterol 13: 432-437.

4. Serour F, Witzling M, Frenkel-Laufer D, Gorenstein A (2008) Intestinal obstruction in an autistic adolescent. Pediatr Emerg Care 24: 688-690.

5. Robles R, Parrilla P, Escamilla C, Lujan JA, Torralba JA, et al. (1994) Gastrointestinal bezoars. Br J Surg 81: 1000-1001.

6. Grimes IC, Spier BJ, Swize LR, Lindstrom MJ, Pfau PR (2013) Predictors of recurrent ingestion of gastrointestinal foreign bodies. Can J Gastroenterol 27: e1-4.

7. Iwamuro M, Okada H, Matsueda K, Inaba T, Kusumoto C, et al. (2015) Review of the diagnosis and management of gastrointestinal bezoars. World J Gastrointest Endosc 7: 336-345.

8. Nasri B, Calin M, Shah A, Gilchrist B (2016) A rare cause of small bowel obstruction due to bezoar in a virgin abdomen. Int J Surg Case Rep19: 144-146.

9. Yakan S, Sirinocak A, Telciler K E, Tekeli M T, Denecli A G (2010) A rare cause of acute abdomen: Small bowel obstruction due to phytobezoar. Ulus Travma Acil Cerrahi Derg 16: 459-463.

10. Ripolles T, Garcia-Aguayo J, Martinez M J, Gil P (2001) Gastrointestinal bezoars: Sonographic and CT characteristics. AJR Am J Roentgenol 177: 65-69. 